\title{
DIAGNOSES FOR PEOPLE WITH HEART DISEASE USING INTERACTIVE MULTIMEDIA
}

\author{
Paryati $^{1 \rrbracket}$ \\ ${ }^{1}$ Informatics Engineering Sciences, Industrial Engineering Faculty, UPN "Veteran" Yogyakarta,+62-485733, Indonesia.
}

\begin{abstract}
Cardiovascular attack (heart) is the death of the heart muscle due to a sudden obstruction of the coronary artery by blood clotting. Wounds on the heart muscle cause chest pain and pressure. If blood flow is not restored again within 20 to 40 minutes, then the death of the heart muscle will no longer begin to occur. The muscle continues to die six to eight hours at a time when the heart attack is completely. Ruptured plaque creates blood clots. If the blood clot is large enough it can close the entire artery resulting in a heart attack. Most cardiovascular diseases are caused by high blood pressure. So the authors made cardiovascular detection research using interactive multimedia with rich internet application method.

The software that supports in the creation of this application is Adobe Flash CS3, Action script as well as Dreamweaver. The methodology system development is multimedia with concept stages, design, material collecting, assembly, testing.

This application aims to disease, causes, various diseases, symptoms, ways of prevention and treatment methods. be utilized as a medium of information and learning for cardiovascular people and for users about the definition of cardiovascular.

Keywords: Multimedia Interactive, Rich Internet Application, Cardiovascular.
\end{abstract}

૫Corresponding author: yaya_upn_cute@yahoo.com 


\section{Introduction}

Cardiovascular disease (heart) is a disease that many people fear. Symptoms and its causes are still difficult to detect and diagnose. Information and knowledge about cardiovascular disease, symptoms, causes, ways of prevention and treatment is still limited. Even the books that talk about the disease are still lacking. Patients with cardiovascular disease and the public need knowledge, information and learning systems about the disease, Even a heart attack is a death of the heart muscle caused by a sudden obstruction of the coronary artery by blood clotting. The coronary artery is a blood vessel that supplies the heart muscle with blood and oxygen. Obstruction of the coronary artery removes blood and oxygen from the heart muscle causing wounds to the heart muscle. Wounds on the heart muscle cause chest pain and chest pressure. If blood flow is not restored again within 20 to 40 minutes, then the death of the heart muscle will no longer begin to occur. The muscle continues to die continuing to die six to eight hours at a time when the heart attack is completely. The cause of the heart attack occurred due to a blockage of the coronary artery after the rupture of the atherosclerosis plaque which is a pile of fatty acids and white blood cells on the wall of the coronary artery that supplies blood to the heart. Ruptured plaque creates blood clots. If the blood clot is large enough it can close the entire artery resulting in a heart attack. Most heart disease is caused by high blood pressure which contributes to hardening of the arteries, high levels of bad cholesterol due to an uncontrolled diet with high levels of saturated fats and trans fats. It adds to the formation of atherosclerosis lesions and eventually arteries that can damage the lining of blood vessels and hinder the transport of oxygen and nutrients to the heart which can lead to a risk of heart attack.

At this time many types of cardiovascular disease discovery. So the authors made research on cardiovascular detection using interactive multimedia with ria method. With the advancement of computer technology can help humans in various fields one of them is interactive multimedia applications 1 .

This application is a learning system that can provide more detailed and in-depth knowledge for heartdisease patients and the general public who need information about heart disease, causes, various heart diseases, symptoms, prevention methods and treatment methods so as to help heart disease patients. The system can also detect and diagnose types of heart disease based on the symptoms experienced by people with heart disease. Treatment based on the identification of diseases experienced by patients who have been studied previously by experts or heart disease doctors, some in the form of advice, advice even encouraged.

The formulation of the problem is how to make cardiovascular detection research using interactive multimedia with Rich Internet Application (RIA) method that can help people with cardiovascular disease and for the general public.

The purpose of this study is to create a learning application that can be utilizedas a medium of information and knowledge for heart disease patients and the general public about the definition of heart disease, causes, various heart diseases, symptoms, ways of prevention and treatment methods that canhelp heart disease patients and the general public who need that information.

Themethod of development of the system used in making and building this research is to follow the methodology of multimedia development with the following stages: concept ( concept), design ( design), collection of materials (material collecting) and the manufacture of programs (assembly) and continued testing programs (testing) 10. In the build and build of this system until the testing stage of the program and application program successfully run according to its function.

The library reviews that support this research include:

1. Creation of Multimedia Applications to Help Geometry Learning. In the study, objects used in the study of problems related to geometry learning[5].

2. Experiential Interactive Multimedia Learning Application Model With Virtual Visualization to Improve Concept Understanding in Electrical Basic Physics Courses of Electricity ( Case Study: Informatics Engineering Majalengka University). In the study, the object relates to the study of Electrical Basic Physics Courses 12 .

The above studies are different from the research thatwasmade bythe authors in making cardiovascular detection research using interactive multimedia with Rich Internet Application (RIA) method. But general many aspects obtained by previous researchers and can provide support for the information required in this study.

\section{Discussion}




\subsection{Concept}

\subsection{Design}

Design is an overview of the software system to be created. The purpose of the design is to give users a more general picture of cardiovascular detection using interactive multimedia with the Rich Internet Application method to be created, and to provide a complete picture of the design as a guide for programmers in building their application system. This design includes navigation structure, flowchart view, storyboard, material collecting.

\subsection{Navigation Structure Design}

The Navigation Structure used in the creation of cardiovascular detection using interactive multimedia with the Rich Internet Application method is the Hierarchical navigation structure. The concept of navigation begins with a single node being the main page and the start page. The first page to be found is the intro page, then it will go to the menu page. The menu has eight submenus namely home, definition, causes, various cardiovascular diseases, symptoms, prevention methods, treatment methods and exiting the system. $\mathrm{P}$ thereis a home page that explains how the heart system works moving on the human body. In the menu the definition explains the meaning of heart disease and explains the learning system of heart disease along with the system of work and how the heart works in the human body. In the menu of causes explaining about the causes of cardiovascular disease that can be caused by several factors including factors age, offspring, smokers, diabetes, hypertension, obesity, lifestyle and high emotions. On the menu of various heart diseases consists of atherosclerosis, IMA, KKJ, GKJ, Ardiomipati, Arrimatia, PJR, Inflammation. On the symptom menu consists of several symptoms that are inflicted including fatigue, chest pain, excessive sweating, headache, nausea, body aches, irregular heartbeat, shortness of breath, swelling when bodily fluids accumulate. Page out contains warnings "yes" and "no" otherwise going back to the menu page 9 .

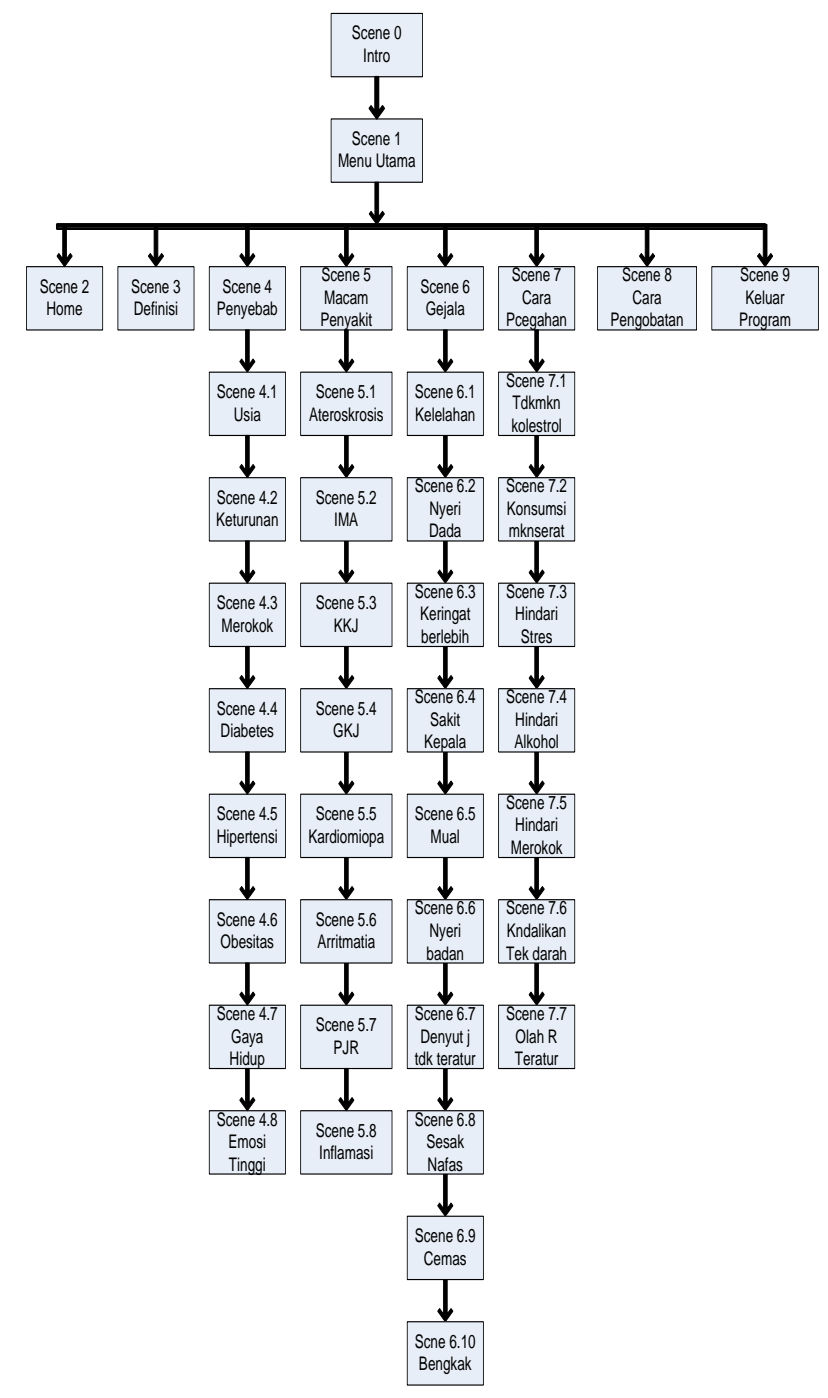

Fig. 1. Navigation Structure.

\subsection{Flowchart View Design}

Flowchart View is a diagram that can give you an overview of the flow of data from one scene to another. In the flowchart view you can see the components contained in the following scenes by transitioning from one scene to another. The relationship between one scene and another is expressed by a line and arrow.

\subsection{Storyboard Design}

The storyboard design contains a visual picture of the design of the scene,the audio used, the duration of each scene, and also the description that explains the scene. The result of this storyboard will be used as a reference in the creation of applications at the implementation stage. 


\subsection{Table Design}

The design of database tables in heart disease applications with RIA technology contains the fields used in each table file. And shows the relationships between tables in each of those database files.

\subsection{Implementation and Discussion}

Once the system is analyzed and designed in detail, the next step is to implementation the system. At this stage displayed interface (interface) cardiovascular detection using interactive multimedia with Rich Internet Application method by using flash. And it will be explained about the hardware and software used in building this system along with pieces of ActionScript programs to connect files to each other.

\subsection{Devices Used}

\subsubsection{Specifications hardware}

The hardware specifications used to build this application are:
a. Processor Intel(R) Celeron(R)
b. CPU N2840 @ 2.16GHz 2.16GHz
c. Memory 2.00 GB RAM
d. $500 \mathrm{~GB}$ hard drive
e. Display 1366 x 768 .

\subsubsection{Specification software}

The software used in building this application is as follows:

Microsoft Windows 10 Home Single Language Operating System 64-bit.

- Programming Languages : ActionScript 2.0 and PHP MySQLi [3].

- Programming and Web Applications : PHP [7] and MySQL [11].

- Graphics Processor : Adobe Flash CS 3 Professional 6.

\subsection{Program Implementation}

In this chapter will be discussed about the implementation of the application program namely the application view, how the application works, so that it can produce the desired output as well as program modules for some processes that occur in the system. Application programs are used as a means of connecting between the user and the computer, so that with the application program the user becomes easier. Here are some examples of the views of each scene that exist in cardiovascular detection using interactive multimedia with the Rich Internet Application method.

\subsubsection{Main menu page view}

The main menu page is the first scene that the user will encounter when entering the app. This main menu contains the home menu, definition, cause, variety, symptoms, prevention, treatment and exit of the system. The following is a view of the main menu page of cardiovascular detection using interactive multimedia with the Rich Internet Application. The main menu page of cardiovascular disease is taken from the reference 9 .

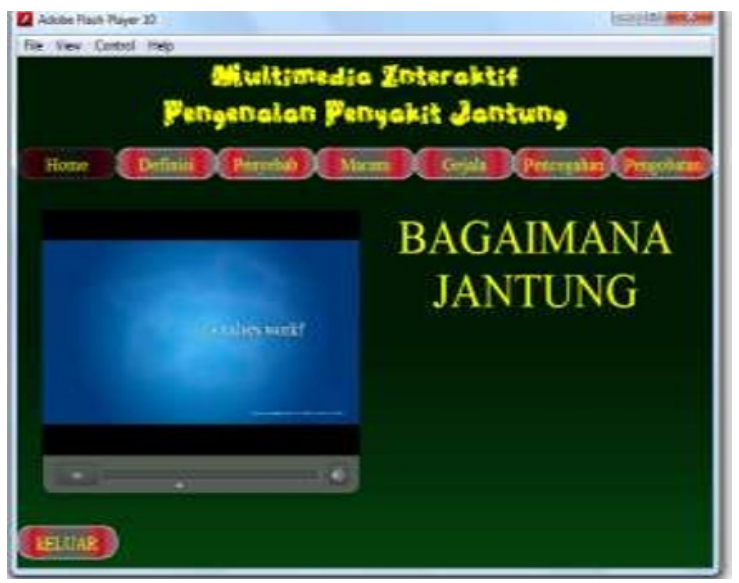

Fig. 2. Main menu page view.

\subsubsection{Home menu page view}

The home menu page is the second scene that the user will encounter when entering this application. Here's a look at the page. This menu contains an explanation of how cardiovascular disease works in the human body 4 .

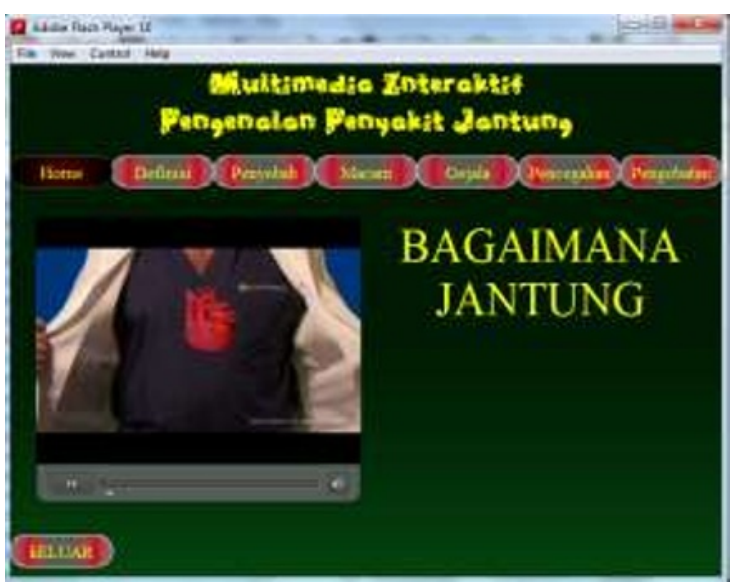

Fig. 3. Home menu page view. 


\subsubsection{Page view definition menu}

The menu page definition of cardiovascular disease is the third scene that the user will encounter when running this application. In this scene contains an explanation of the understanding of cardiovascular disease and learning as well as knowledge of cardiovascular disease. Here's a look at the menu page. The definition of cardiovascular disease is taken from reference 2 .

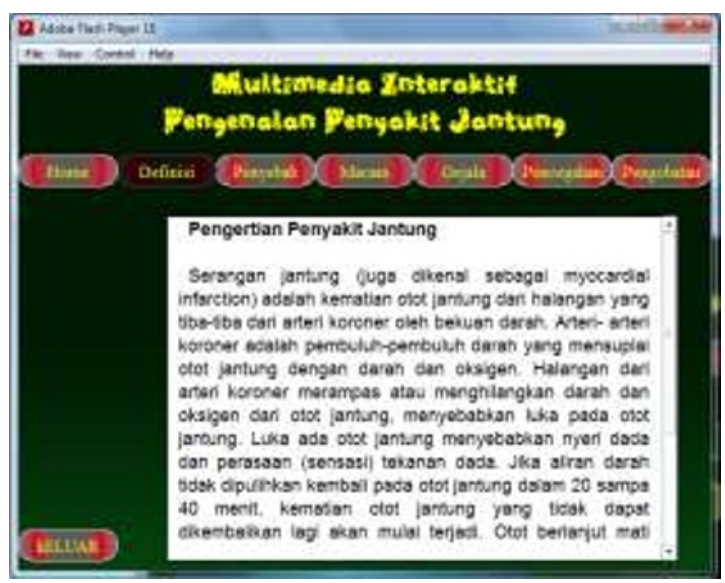

Fig. 4. Page view definition menu.

\subsubsection{Cause menu page view}

The menu page of cardiovascular disease that can attack the human body is the fourth scene that the user will encounter when running this application. In this scene contains various sub menus contained in the application consisting of eight sub menus, among others: sub menu age, offspring, smokers, diabetes, hypertension, obesity, inappropriate lifestyle, high emotional level. Here's a look at the menu page. The cause of cardiovascular disease is taken from reference 8 .

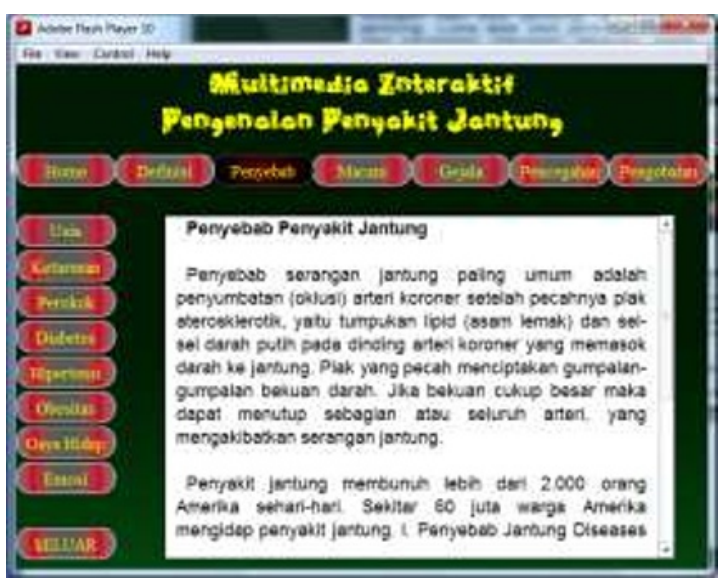

Fig. 5. Cause menu page view.

\subsubsection{Sort menu page view}

In the scene of various heart diseases it contains various sub menus contained in the application. The menu page of various heart disease is the fifth scene that users will encounter when running this application consisting of eight sub menus including: atherosclorosis, IMA, KKJ, GKJ, cardiomyopathy, arritmatia, PJR, Inflammation. The following is a view of the menu page of various heart diseases from cardiovascular detection using interactive multimedia with rich internet application method. Various diseases are taken from references 4 .

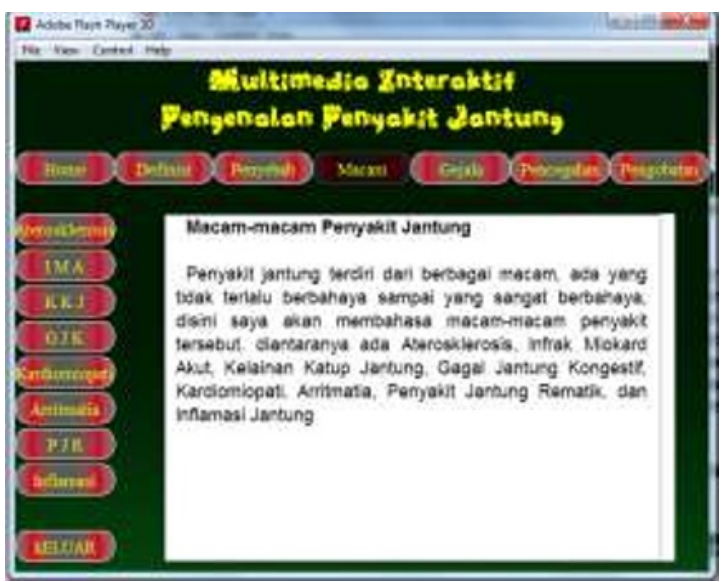

Fig. 6. Sort menu page view.

\subsubsection{Symptosm menu page view}

The menu page of symptoms of cardiovascular disease is the sixth scene that the user will encounter when running this application. In this scene contains various menus of symptoms of heart disease contained in the application. The following is a view of the symptom menu page of cardiovascular detection using interactive multimedia with the Rich Internet Application method. The symptoms of heart disease are taken from reference 8 . 


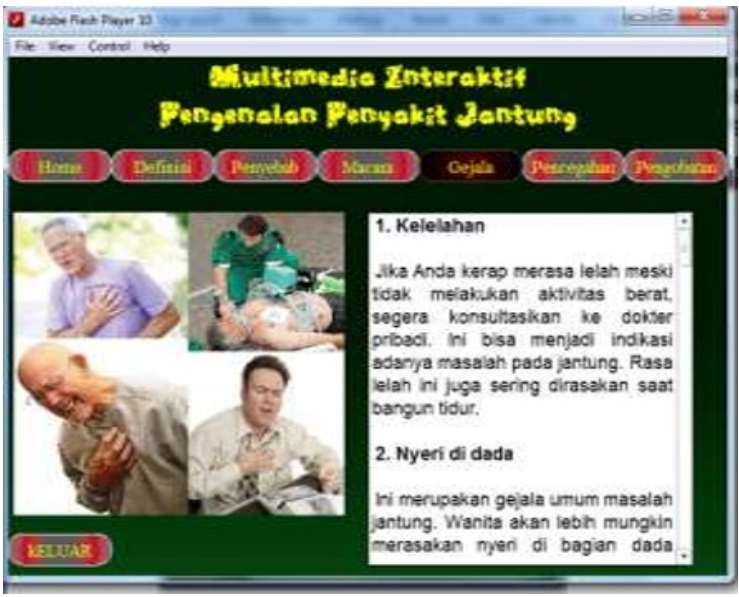

Fig. 7. Symptoms menu page view.

\subsubsection{Prevention menu page view}

The heart disease prevention menu page is the seventh scene that users will encounter when running this application. In this scene contains various menus contained in the application. Here's a look at the menu page. The way of prevention of heart disease is taken from reference 8 .

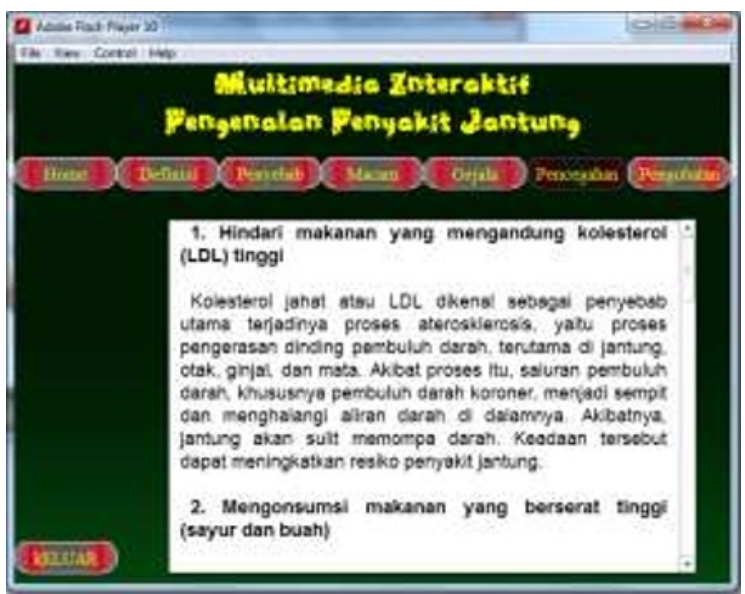

Fig. 8. Prevention menu page view.

\subsubsection{Treatment menu page view}

The cardiovascular disease treatment menu page is the eighth scene that users will encounter when running this application. In this scene contains various menus contained in the application. Here's a look at the menu page. The treatment of cardiovascular disease is taken from reference 8.

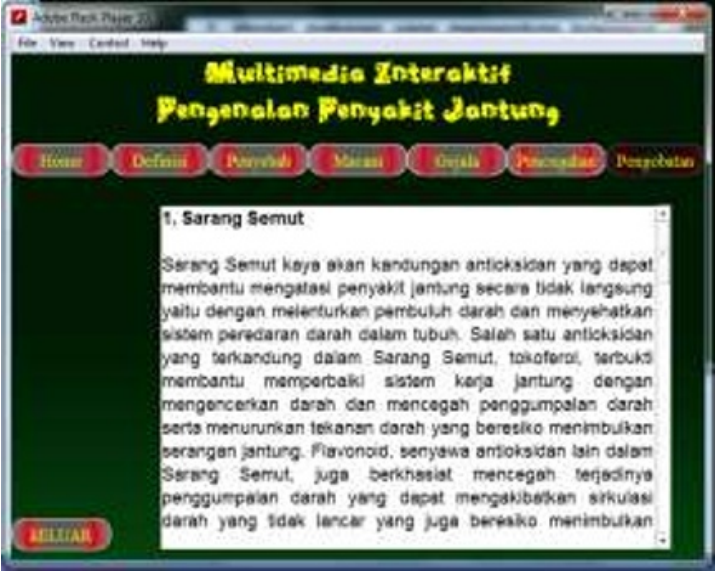

Fig. 9. Treatment menu page view.

All examples of gambar 1.Navigation Structure taken from the reference source of the book 9 .

\section{Conclusion}

\subsection{Conclusion}

Based on the results of this study, the following conclusions can be drawn:

a. Provide information to the user about cardiovascular disease suffered, initial diagnosis based on the symptoms given.

b. Help users understand and obtain information about cardiovascular disease types .

c. Assisting the user in identifying cardiovascular disease early, through the processing of symptom data, so that further treatment of the disease can be done quickly.

d. Provide information to the user about how treatment can be done and the healing therapy.

e. The data contained in the system can be updated or added as needed.

\subsection{Suggestion}

This application can be developed further to be more perfect in terms of :

a. You can add more program menus to make it more complete.b. Kinds of diseases were inputted again so that more data.

b. Data curing methods can be added with the latest findings so that they are complete.

c. Data for program applications can be inputted again so that the data reference is more complete.

d.The method of treatment can also be supplemented with the latest findings. 


\section{References}

1. Darma, S. Jarot, Smart books master multimedia, PT.Transmedia, Jakarta (2019)

2. D. Hawari, Coronary Heart Disease, Faculty of Medicine, University Indonesia, Jakarta, (2015)

3. L. Hakim, PHP master core secret mySQLi improved, CV. Lokomedia, Yogyakarta, (2015)

4. Kabo, Z. Peter, Coronary heart disease or scientific processes, Faculty of Medicine, University Indonesia, Jakarta 2015

5. Lisana, Making multimedia application to help learninggGeometry, Proceeding Snasti STIKOM Surabaya, page 456-460, 2018

6. MADCOMS, Proficient in 7 days adob flash CS6, Andi Offset, Yogyakarta, 2018

7. B. Nugroho, Dynamic web programming application with $P H P$ and MySQL, Gava Media, Yogyakarta, 2015

8. I. Soeharto, Coronary heart disease and heart attack, prevention, cure and rehabilitation, Faculty of Medicine, University Indonesia, Jakarta, 2015

9. A. H. Sutopo, Multimedia interactive with flash, Graha Ilmu, Yogyakarta, 2018

10. M. Suyanto, Multimedia tools to increase competitive advantage, Andi Offset, Yogyakarta, 2018

11. Sutarman, Build web applications with PHP and MySQL, Graha Ilmu, Yogyakarta, 2018

12. T. Wahyuni, Experiential interactive multimedia learning application model with virtual visualization to improve understanding of concepts in basic physicscCourses of electricity case study:informatics engineering, Majalengka University, in the study, the object relates to the study of Electrical Basic Physics Courses. In Proceedings of the National Congress on the object relates to the study of Electrical Basic Physics Courses, Semnasif, page 292-307, 22-23 October 2016, UPN "Veteran" Yogyakarta, 2015 